\title{
CARDIOMYOPATHY AS ONE OF THE LESS FREQUENT MANIFESTATIONS OF TAKAYASU'S ARTERITIS
}

\author{
Lucie Horáková', Radek Pudil', Zbyněk Hrnčířr', Jaroslav Vižd'a ${ }^{3}$
}

Charles University in Prague, Faculty of Medicine and University Hospital Hradec Králové, Czech Republic: 1st Department of Medicine ${ }^{1}$, Rheumatological Unit - 2nd Department of Medicine ${ }^{2}$, Department of Nuclear Medicine ${ }^{3}$

Summary: The authors present the case of a young woman with newly diagnosed Takayasu's arteritis. This woman, with arterial hypertension, was investigated for the unspecific symptoms at the beginning. Afterwards, the transthoracic echocardiography showed dysfunction of the left ventricle and the abdominal sonography showed a stenosis of the right renal artery. PET/CT scan showed chronic modification after inflammatory processes on the wall of the thoracic and abdominal aorta. This case report should be instructive to other clinicians and refers to the necessity to remember this rare disease in our country too.

Key words: Cardiomyopathy; PET/CT scan; Takayasu arteritis

\section{Introduction}

Takayasu's arteritis is a chronic inflammatory disease of unknown etiology that primarily involves the aorta, its major branches and the pulmonary arteries. The pathology consists of granulomatous changes in the media and adventitia (medial degeneration, adventitial fibrosis) (1). Takayasu arteritis is more common in Asian persons and females are more frequently affected $(97 \%)$. The median age at disease onset is 25 years (2). The diagnosis of Takayasu arteritis often is delayed for months because many patients manifest nonspecific symptoms. At the end the changes in the wall of the arteries lead to clinical manifestation of ischemia. There are four possible treatments: corticosteroids, cytotoxic therapy, percutaneous transluminal angioplasty (PTCA) and surgery. The 5-year mortality rate from some studies is nearly $35 \%$ and the $23 \%$ patients never achieve remission (3).

\section{Case report}

A 20-year-old woman with a short history of arterial hypertension was admitted for a newly diagnosed cardiomyopathy of unknown etiology. Six months prior to her admission, this woman was examined for elevated erythrocyte sedimentation, C-reactive protein, leukocytosis and other non-specific symptoms such as fever and fatigue. On admission, the physical examination revealed a blood pressure of the $180 / 110 \mathrm{mmHg}$, a pulse rate of $70 / \mathrm{min}$ and systolic murmur with the maximum on the position of the mitral valve; no abnormalities were found on the lung, abdomen or skin. The laboratory revealed increased inflammation markers (leukocyte count $9.97 \times 10^{3} / \mu \mathrm{L}$, erythrocyte sedimentation rate 21/34, C-reactive protein $24 \mathrm{mg} / \mathrm{L}$ ). Electrolytes, urea and creatinine concentrations were within normal limits. The electrocardiogram revealed sinus rhythm. The echocardiogram showed systolic dysfunction of the left ventricle (ejection fraction 30\%). There were no local disorders of movement (the maximum diameter of the left ventricle was $49 \mathrm{~mm}$ ) and no signs of hypertrophy of the chambers. The echocardiogram showed medium mitral valve regurgitation (restriction of the posterior leaflet and prolapse of the anterior leaflet).

The magnetic resonance imaging of the heart confirmed the results of the transthoracic echocardiography and did not show any other structural abnormalities. The coronary arteries were without any stenosis on the coronary angiography. The abdominal sonography showed hypotrophy of the right kidney and renal radiography confirmed its severe dysfunction (function $<1 \%$ ). The abdominal magnetic resonance imaging showed a critical stenosis of the long part of the trunk of the right renal artery (Fig. 1-2), hypotrophy of the right kidney and local increase of the wall as well as stenosis of the subrenal aorta. These findings led us to the suspicion of a large-vessel vasculitis. We found antinuclear factor (ANF) whose concentration was over the normal limits. A subsequent PET/CT scan with 18F-fluorodeoxyglukose (FDG) showed chronic modification following the inflammatory process on the wall of the subrenal aorta and on the right renal artery as well as showing the concentration of active inflammation on the wall of the thoracic aorta and the right subclavian artery (Fig. 3-5). After these findings corticosteroids therapy was started. The arterial hypertension was compensated by a combination of beta-blockers, angiotensin-converting enzyme inhibitors and calcium-blockers. 

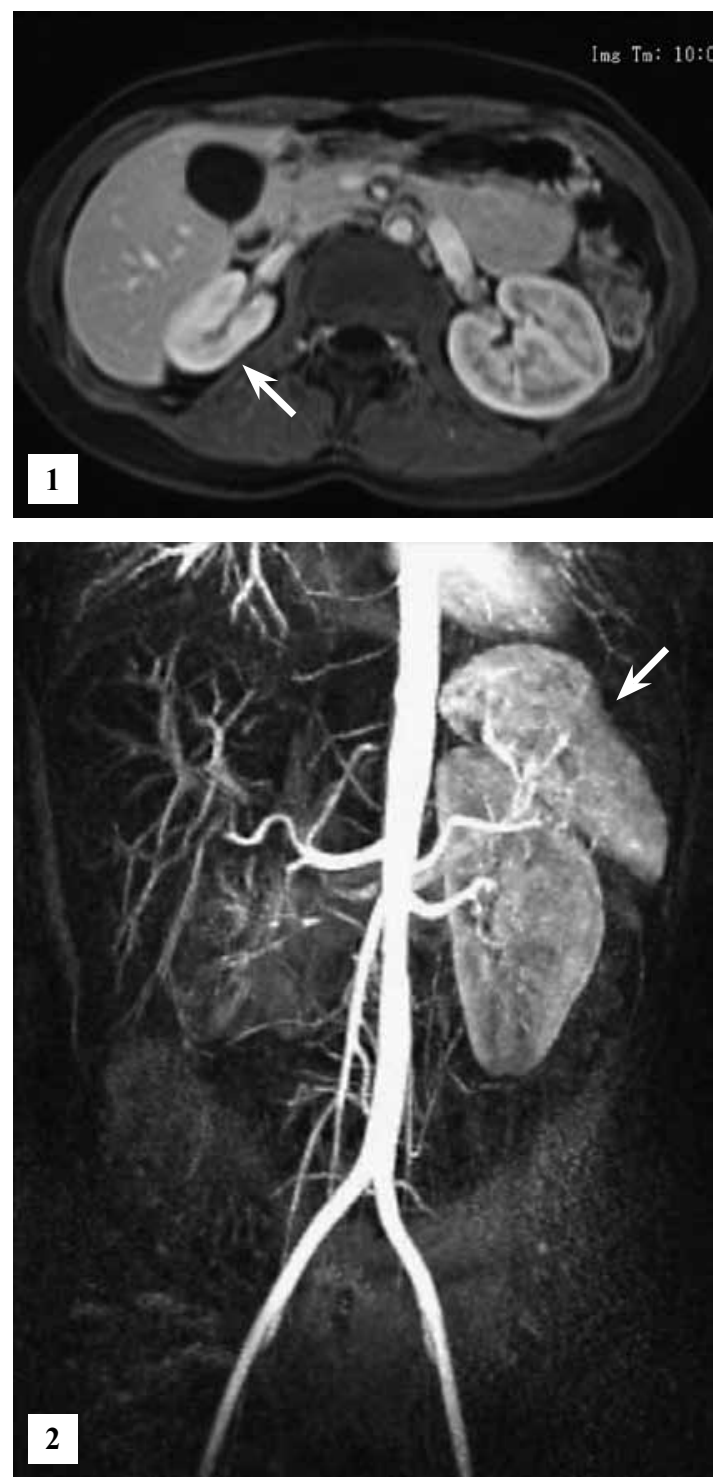

Fig. 1-2: The abdominal magnetic resonance imaging demonstrates the stenosis of the long part of the trunk of the right renal artery, the hypotrophy of right kidney and the stenosis of the subrenal aorta

\section{Discussion}

Takayasu's arteritis (TA) is a granulomatous systemic idiopathic disease of the aorta and its primary branches that may lead to segmental stenosis, occlusion, dilatation or aneurysm formation $(1,4,5)$. The incidence of the disease is higher in Asia and the disease affects more frequently young women. During the past few decades, patients with TA have been increasingly recognized in Africa, Western Europe and North America (2). The estimated incidence rate is 2.6 cases per million persons per year. Many patients manifest for a long time with nonspecific symptoms (fa-
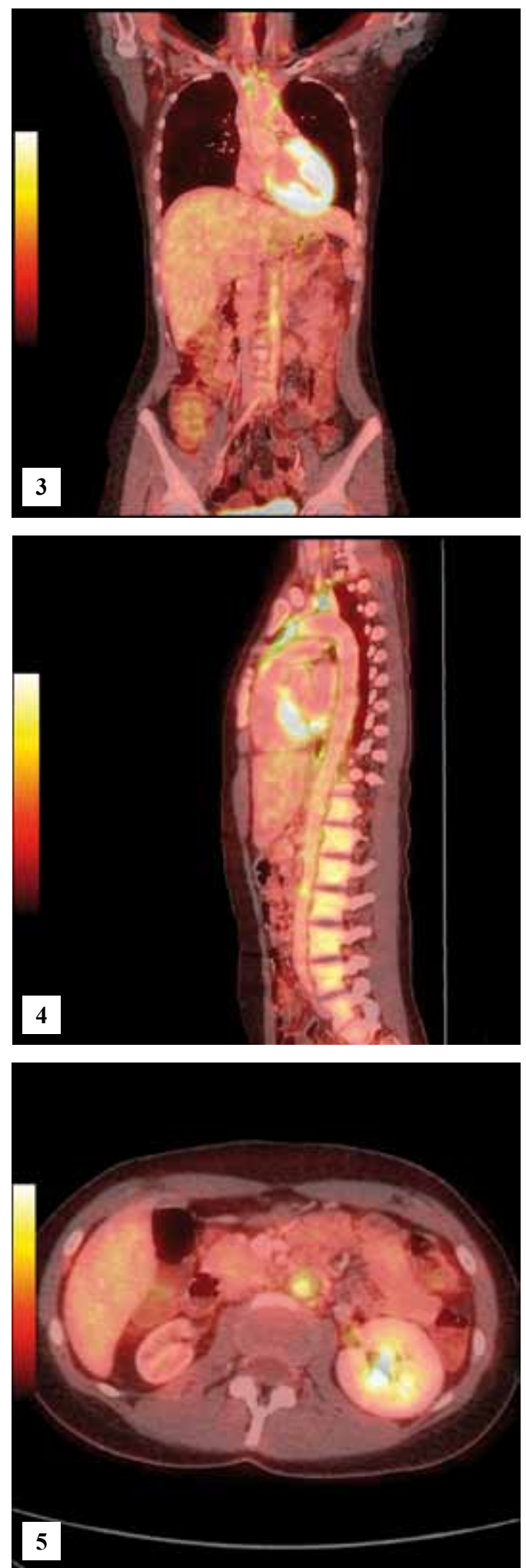

Fig. 3-5: Fused PET/CT images demonstrate abnormally increased FDG uptake in the increase of the wall of the subrenal aorta and along the wall of the thoracic aorta and the right subclavian artery 
tigue, malaise, weight loss, night sweats, fever, arthralgias, myalgias or anemia). Vessel wall inflammation may cause organ ischemia due to arterial stenosis or occlusion (in rare cases we see vascular dissection or rupture) leading to clinical manifestations. Inflammatory cell infiltration suggests that cell-mediated autoimmunity plays an important role in the pathogenesis of TA (6). The patients often have arterial hypertension, which is frequently associated with renal artery stenosis. The laboratory data are typical for generalized inflammation (slight leukocytosis and mild anemia, elevation of the C-reactive protein, thrombocytosis and elevation of erythrocyte sedimentation). Other laboratory data (antinuclear antibodies, rheumatoid factor, antineutrophil cytoplasmic antibodies, etc.) are very often within normal limits. The disease can affect the cardiovascular system (cardiomyopathy, valvular disease, aortic root disease, coronary disease or constrictive pericarditis).

In our case the woman had nonspecific symptoms (fatigue, fever), elevated inflammation markers, arterial hypertension associated with renal artery stenosis, systolic dysfunction of the left ventricle (ejection fraction 30\%) and medium regurgitation of mitral valve. Some radiographic methods can be useful in diagnosing TA. Arteriography is the standard for detecting vessel disease. Color Doppler sonography can show stenosis of the arteries. Transthoracic echocardiography can reveal cardiac disorders. Currently, magnetic resonance imaging (MR) is a very useful noninvasive examination. It reveals vessel wall oedema in $94 \%$ of the cases (7). PET/CT scan with $18 \mathrm{~F}$-fluorodeoxyglukose (FDG) is a new method able to reveal deposits of inflammation in vessel walls. Laboratory and radiographic investigations in TA are initially useful for the diagnosis and are necessary for assessing disease activity.

The American College of Rheumatology (ACR) provides criteria that best classify patients with TA in comparison with a control group of patients with other forms of vasculitis. For the classification of TA, ACR requires three of the following six criteria: age at the disease onset $<40$ year, claudication extremities, decreased brachial artery pressure, blood pressure difference $>10 \mathrm{mmHg}$, a bruit over the subclavian arteries or aorta and abnormal arteriogram (arteriographic evidence of narrowing or occlusion of the entire aorta, its primary branches, or large arteries in the proximal upper or lower extremities) (1). The presence of three or more of these six criteria demonstrates a sensitivity of $90.5 \%$ and a specificity of $97.8 \%$. These criteria are not met in the early stages of the disease.

Corticosteroids in high doses are the primary treatment recommended for TA. Cytotoxic therapy is recommended for patients that are resistant to glucocorticoid treatment. The overwhelming majority of experience is with the use of methotrexate (MTX) and clophosphamide. New medications are, however, being tested. Mycophenolate mofetil may be an alternative to steroids and cytotoxic agents (6) or anti-TNF therapy (tumor necrosis factor is important in the formation of the granulomas) (8). Anti-TNF therapy may be a useful adjunct to glucocorticoids. Surgical or percutaneous transluminal angioplasty therapy is important in the revascularization of stenosed or occluded vessels which produce significant ischemia.

Takayasu's arteritis is associated with young women and it is important to consider that incidental gravidity should be carried out by $\mathrm{C}$-section.

The authors of this case report stress the need to bear this disease in mind in our country as well.

\section{References}

1. Arrend WP, Michel BA et al. The American college of rheumatology 1990 criteria for the classification of Takayasu arteritis. Arthritis and Rheumatism. 1990, Vol. $33,1129-1134$

2. Kerr GS, Hallahan CW et al. Takayasu arteritis. Ann Intern Med. 1994, 120, 919-929.

3. Langford CA, Hochberg MC et al. Rheumatology. Third edition. 2003, 1657-1663.

4. Schmidt WA, Nerenheim A et al. Diagnosis of early Takayasu arteritis with sonography. Rheumatology. 2002, 41, 496-502.

5. Hoffamn GS, Merkel PA et al. Anti-tumor necrosis factor therapy in patients with difficult to treat Takayasu arteritis. Arthritis and Rheumatism. 2004, 50, 2296-2304.

6. Daina E, Schieppati A et al. Mycophenolate Mofetil for the treatment of Takayasu arteritis: report of three cases. Ann Intern. Med. 1999, 130, 422-426.

7. Tso E, Flamm SD et al. Utility and limitations of magnetic resonance imaging in diagnosis and treatment. Arthritis and Rheumatism. 2002, Vol. 46, 1634-1642.

8. Hoffman GS, Merkel PS et al. Anti-Tumor necrosis factor therapy in patients with difficult to treat Takayasu arteritis. Arthritis and Rheumatism. 2004, Vol. 50, 2296-2304.

Received: $27 / 04 / 2011$

Corresponding author:

Accepted in revised form: 27/10/2011

Lucie Horáková, M.D., University Hospital Hradec Králové, Sokolská 581, 50005 Hradec Králové, Czech Republic, 1st Department of Medicine; e-mail: lucka.horakova@email.cz 